\title{
Upshot of Marangoni nanofluid thin film flow under the influence of an inclined magnetic field
}

\author{
Muhammad Ramzan and Hina Gul \\ Department of Computer Science, Bahria University, Islamabad Campus, Islamabad, 44000, Pakistan
}

Received: 13 June 2021, Accepted: 16 June 2021

Published online: 8 July 2021.

\begin{abstract}
A numerical simulation is conducted for the applied inclined magnetic field on a two dimensional electrically conducting unsteady Marangoni nanofluid thin-film flow past a horizontal extending sheet. The composite of nanofluid comprises Copper nanoparticles and kerosene oil base fluid. The envisioned flow is also affected by the non-uniform source/sink and viscous dissipation. The succor of the Shooting technique amalgamated with the Fehlberg Runge-Kutta computational scheme is secured for the solution of a highly nonlinear system of equations. The significant impacts of the prominent arising parameters versus involved fields are examined through graphs. The outcomes show that the fluid velocity of the thin film nanofluid flow is curbed for a strong magnetic field nevertheless an opposing trend is witnessed in case of the liquid temperature. Furthermore, the rate of heat flux is escalated versus estimates of nanoparticle volume fraction and time-dependent parameter.
\end{abstract}

Keywords: Marangoni effect, Nanofluid thin film flow; Numerical solution; Non-uniform source/sink.

\section{Introduction}

Nanofluids, an evolving field of engineering have trapped the eye of numerous researchers who were looking at the ways to improve the efficiency of cooling in industry. Nanoliquid is a fluid that is made by propagation of strong particles with sizes less than $100 \mathrm{~nm}$ in fluids. A nanofluid low thermal conductivity is one of its striking parameters that can restrain the performance of transfer of heat. Due to their short heat transfer properties, normal heat transport fluids such as $\mathrm{H}_{2} \mathrm{O}$ and $\left(\mathrm{CH}_{2} \mathrm{OH}\right)_{2}$, and motor oil have limited heat transfer capabilities and are therefore unable to meet today's cooling requirements. Suspended nanoparticles can improve the fluid flow and heat transfer features of the base liquid. Essential studies must be conducted before extensive application for nanofluids can be encountered. Sheikholeslami et al. [1] examined MHD nanofluid heat convection behavior with an inclined L shape enclosure. They discussed the behavior of different parameters on streamlines, isotherm, and heat lines. Investigators have given special consideration to thin film flows in the last few decades. From environment to engineering i.e., coating streams, films of the tear in a human eye, constant forming, striating of foodstuff, the use of a membrane in biophysics, and in several other manufacturing trends, thin-liquid films play a pivotal role. The leading theoretical study on the flow of the thin film is proposed by Emslie et al. [2]. The balance between viscous and centrifugal forces is considered in this investigation during the disk rotating process. The key finding of this investigation is that the thinning characteristics of the thin film are upheld during the whole process. The difference in the fluid surface tension with respect to the concentration or temperature is documented as the Marangoni impacts. This inconsistency in surface tension results in convective transport of Marangoni convection and liquid. The impact of Marangoni convection may be witnessed in material science, aerospace, thin liquid films, silicon melts, and chemical engineering. Researchers have shown a great interest in this special effect. Radiative properties of Marangoni and hybrid nanoliquid flow past over a porous surface with magnetic field (inclined) analyzed by Mdallal et al. [3]. They utilize the Laplace technique to achieve the analytical solution to the problem. Some more published 
articles regarding thin-film flow with an inclined magnetic field are can be found in references [4-6]. The aforementioned studies disclosed that no study so far carried out discussing the Marangoni effects in a nanofluid thin film flow influence by an inclined magnetic field with a non-uniform heat source/sink. The nanofluid is comprising the copper nanoparticles immersed into the Kerosene oil. Thus, our aim here is to analyze the inclined magnetic field effect on time-dependent finite nanoliquid thin film flow with heat transfer and viscous dissipation. Influences of noticeable parameters on fluid velocity and energy profiles are portrayed via graphical illustrations. By adopting the RK-4 shooting technique, the numerical results of the current work is obtained. The skin friction coefficient and heat transfer rate are estimated numerically for distinct parameters.

\section{Mathematical modeling}

Let us examine a 2D unsteady, incompressible nano-fluid thin-film flow with non-uniform thickness. An electrically conducted fluid is practiced as a continuous magnetic field. Base fluid Kerosene oil alongside suspended copper nanoparticles is assumed. The impact of thermal stratification and viscous dissipation is also considered. In the film, liquid motion arises initially due to stretchiness of the elastic sheet. Supposing $T_{0}$ and $T_{\text {ref }}$ slit energy and constant reference energy respectively and the wall temperature is $T_{s}=T_{0}-T_{r e f}\left(\frac{b x^{2}}{2 v_{F}}\right)(1-a t)^{\frac{-3}{2}}$ with $0 \leqslant T_{r e f} \leqslant T_{0}$. In Fig.1, a non-conventional inclined magnetic field is displayed vertically. The current magnetic field performs at as a transversal magnetic field.

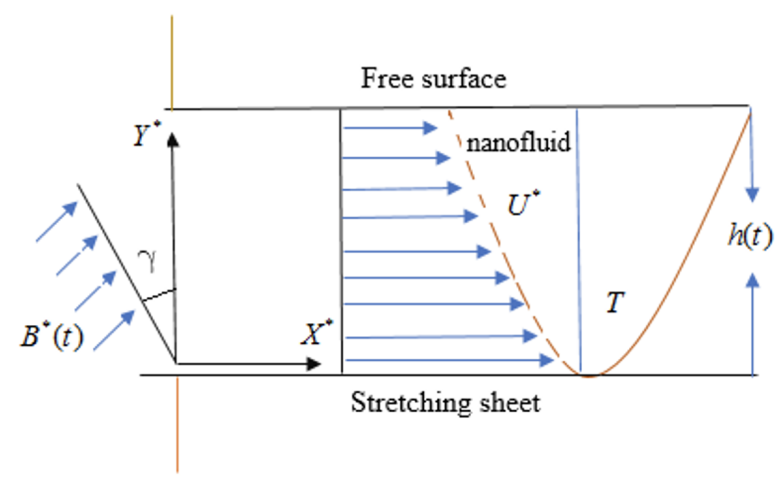

Fig. 1: Physical framework

The governing equations based upon the above Supposition comprise:

$$
\begin{gathered}
U^{*}{ }_{Y^{*}}+U^{*} X^{*}=0, \\
\rho_{N F}\left(U^{*}{ }_{t}+V^{*} U^{*}{ }^{*}+U^{*} U^{*}{ }^{*}=\mu_{N F}\left(U^{*} Y^{*} Y^{*}\right)-\sigma_{N F} B^{* 2}(t) \sin ^{2}(\gamma) U^{*}\right. \\
T_{t}+U^{*} T_{X^{*}}+V^{*} T_{Y^{*}}=\frac{k_{N F}}{(\rho c)_{N F}} T_{Y^{*} Y^{*}}+\frac{k_{F} U_{w}}{x v_{F}\left(\rho c_{p}\right)_{N F}}\left\{A\left(T_{S}-T_{0}\right) f^{\prime}+B\left(T-T_{0}\right)\right\}+\frac{\mu_{N F}}{(\rho c)_{N F}}\left(U^{*}{ }^{*}\right)^{2}
\end{gathered}
$$


Where;

$$
\begin{array}{r}
\rho_{N F}=(1-\varphi) \rho_{F}+\varphi \rho_{s}, \mu_{N F}=\frac{\mu_{F}}{(1-\varphi)^{2.5}} \\
\alpha_{N F}=\frac{k^{*}{ }_{N F}}{(\rho c)_{N F}}, \rho_{N F}=(1-\varphi) \rho_{F}+\varphi \rho_{s} \\
k_{N F}^{*}=k_{F}^{*}\left(\frac{k^{*}{ }_{s}+2 k^{*}{ }_{F}-2 \varphi\left(k^{*}{ }_{F}-k^{*}\right)}{k^{*}{ }_{s}+2 k^{*}+\varphi\left(k^{*}-k^{*}\right)}\right) \\
\sigma_{N F}=\left[1+\frac{3\left(\frac{\sigma_{s}}{\sigma_{F}}-1\right) \varphi}{\left(\frac{\sigma_{s}}{\sigma_{F}}+2\right)-\left(\frac{\sigma_{s}}{\sigma_{F}}-1\right) \varphi}\right] \varphi_{F}
\end{array}
$$

Alongside boundary constraints

$$
\begin{gathered}
\frac{\mu_{N F}}{\mu_{F}} U^{*}{ }_{Y}=T_{X}, V^{*}=0, W^{*}=0,-k T_{Y^{*}}=h_{f}\left(T_{s}-T\right), \\
\text { at } Y^{*}=0 \\
U^{*}{ }^{*}=0, V^{*}=\frac{d h}{d t}, T=T_{0}, \\
\text { at } Y^{*}=h(t) .
\end{gathered}
$$

Now, we introduce the similarity transformations for the above system as

$$
\begin{gathered}
\psi=\beta^{*}\left(\frac{v_{F} b}{1-a t}\right)^{1 / 2} X^{*} f(\eta), T=T_{0}-T_{r e f}\left(\frac{b x^{2}}{2 v_{F}}\right) \frac{\theta(\eta)}{(1-a t)^{\frac{3}{2}}} \\
\eta=\frac{1}{\beta^{*}}\left(\frac{b}{v_{F}(1-a t)}\right)^{1 / 2} Y^{*}
\end{gathered}
$$

Using equations (7), equations (1)-(3) become

$$
\begin{gathered}
\varepsilon_{1} f^{\prime \prime \prime}-\beta^{* 2}\left(f^{\prime 2}+S\left(f^{\prime}+\frac{\eta}{2} f^{\prime \prime}\right)-f f^{\prime \prime}+\varepsilon_{2} M f^{\prime} \sin ^{2}(\gamma)\right)=0, \\
\frac{\varepsilon_{3}}{\operatorname{Pr}} \theta^{\prime \prime}-\beta^{* 2}\left[\frac{S}{2}\left(3 \theta+\eta \theta^{\prime}\right)+2 f^{\prime} \theta-f \theta^{\prime}-A f^{\prime}-B \theta\right]+Q \theta+M E c S f^{\prime \prime 2}=0, \\
f^{\prime \prime}(0)=(1-\varphi)^{2.5} M a \theta^{\prime}(0), f(0)=0, \\
\theta^{\prime}(0)=-B i(1-\theta), f(1)=\frac{S}{2}, f^{\prime \prime}(1)=0, \theta(1)=0,
\end{gathered}
$$

Where

$$
\begin{gathered}
\varepsilon_{1}=\left[(1-\varphi)+\varphi \frac{\left(\rho c_{p}\right)_{s}}{\left(\rho c_{p}\right)_{F}}\right], \varepsilon_{2}=\left[(1-\varphi)+\varphi \frac{\rho_{s}}{\rho_{F}}\right](1-\varphi)^{2.5}, \\
\varepsilon_{3}=\frac{k^{*}{ }_{N F}}{k^{*} F}=\left(\frac{k^{*}{ }_{s}+2 k^{*} F-2 \varphi\left(k_{F}^{*}-k^{*}\right)}{k^{*}{ }_{s}+2 k^{*}+\varphi\left(k^{*}{ }_{F}-k^{*}\right)}\right) . \\
\operatorname{Pr}=\frac{v_{f}}{\alpha_{f}}, S=\frac{a}{b}, M=\frac{\sigma_{f} B_{0}^{2}}{\rho_{f} b}, \\
E c=\frac{b}{\left(c_{p}\right)_{f}\left(T_{s}-T_{0}\right)}, M a=\sqrt{b v_{f}} T_{r e f},
\end{gathered}
$$


The drag surface and Local Nusselt number is

$$
\begin{gathered}
C_{F}=\frac{2 \mu_{N F}}{\rho_{F} U_{w}^{2}}\left(\frac{\partial u^{*}}{\partial Y^{*}}\right)_{Y^{*}=0}=\frac{1}{\beta^{*}} \frac{2}{(1-\varphi)^{2.5}} \operatorname{Re}_{X^{*}}^{-1 / 2} f^{\prime \prime}(0), \\
N u=\frac{-X^{*} k_{N F}}{k^{*}{ }_{F}\left(T_{1}-T_{0}\right)}\left(\frac{\partial T}{\partial Y^{*}}\right)_{Y^{*}=0}=-\frac{k^{*}{ }_{N F}}{k^{*} F} \frac{1}{\beta^{*}} \operatorname{Re}_{X^{*}}^{1 / 2} \theta^{\prime}(0) .
\end{gathered}
$$

Which implies:

$$
C_{F} \operatorname{Re}_{X}^{1 / 2}=\frac{1}{\beta^{*}} \frac{2}{(1-\varphi)^{2.5}} f^{\prime \prime}(0), N u R e_{X^{*}}^{-1 / 2}=-\frac{k^{*} N F}{k^{*} F} \frac{1}{\beta^{*}} \theta^{\prime}(0),
$$

\section{Graphical results with discussion}

This portion is committed to study the outcomes of prominent parameters on specific distributions i.e., velocity and temperature distributions. The impact of the magnetic field $(M)$ against a similarity variable $(\eta)$ on velocity is discussed in Figure 2. Fig 2 demonstrates that increment in $M$ leads to the reduction in velocity profile because at some point in nanofluid, an enhancement in the intensity of the magnetic field causes resistance in fluid motion due to strong forces like Lorentz which reduces the movement of the fluid. Fig 3 is sketched to show the variations of the unsteadiness parameters $(S)$ on velocity field. It is witnessed that fluid velocity reduces for higher estimates of $S$. Physically, buoyancy forces are produced in the fluid due to unsteadiness which acts on the nanofluid and slows down its motion. Thus, a decline in heat transfer from sheet to liquid is witnessed which causes decay in the fluid temperature. The effect of $S$ (unsteadiness parameters) on the temperature profile is portrayed in Fig 4. It can be observed that an augmentation in $S$ causes an increment in temperature distribution. Physically, unsteadiness parameter $\mathrm{S}$ has a direct relation with the fluid temperature. The impact of Biot number $(B i)$ on dimensionless temperature can be seen in Fig 5 . The temperature along with thermal boundary layer thickness fall when we strengthen the values of $\mathrm{Bi}$. It is shown that the temperature field upsurges quickly near the boundary with rise in the Biot number. It is also examined that the convective heating of the sheet is increases as Biot number increases. Fig 6 portray the impact of the Marangoni number $(M a)$ on the velocity profile. Velocity and its layer thickness enlarged for larger values of $M a$. Moreover, the velocity layer thickness meaningfully varies at the surface $(\eta=0)$ for greaterMa. It is due to the surface tension generated by thermo solutal gradients. Tables 1 and 2 portrays different variations of the skin friction coefficient and local heat transfer rate for different estimates of emerging parameters.

Table 1: The tabulation of numerical results for Drag force $C_{f} R e_{x}^{1 / 2}$ against the distinct estimates of rising parameters.

\begin{tabular}{|l|l|l|}
\hline$M$ & $S$ & $C_{f} \operatorname{Re}_{x}^{1 / 2}$ \\
\hline 0.5 & & 0.0451 \\
\hline 1.0 & & 0.0450 \\
\hline 1.5 & & 0.0449 \\
\hline 2.0 & & 0.0448 \\
\hline & 0.5 & 0.0452 \\
\hline & 0.8 & 0.0451 \\
\hline & 1.2 & 0.0450 \\
\hline & 1.8 & 0.0449 \\
\hline & 0.5 & 0.0452 \\
\hline
\end{tabular}




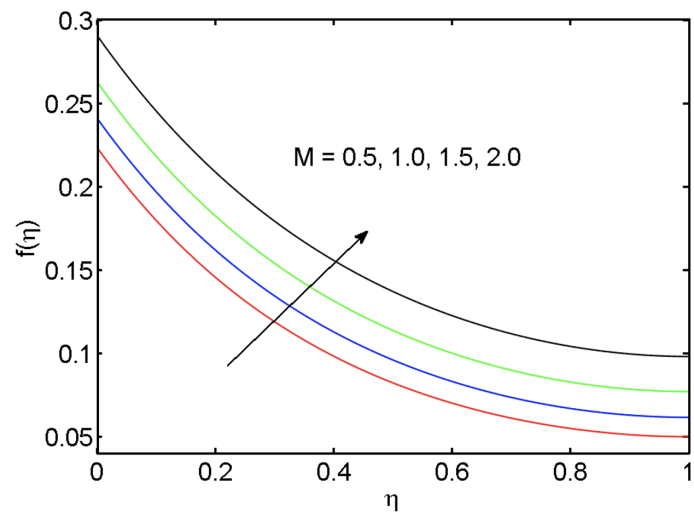

Fig. 2: Variations $M$ to $f^{\prime}$

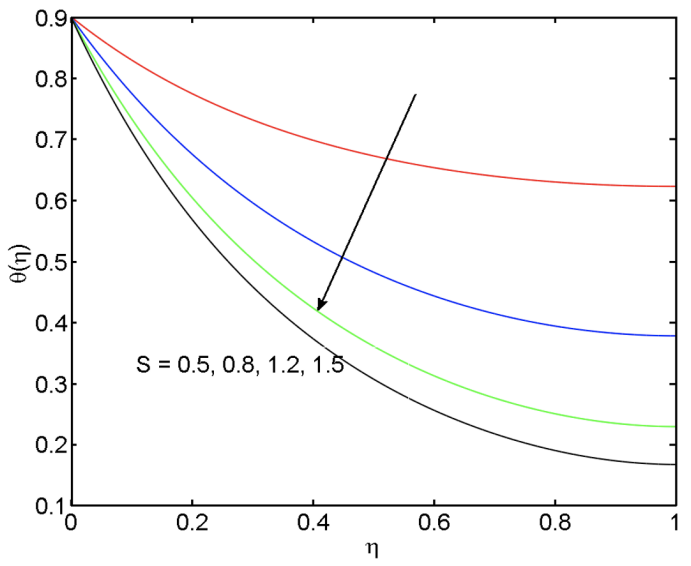

Fig. 4: Variations $S$ to $\theta$

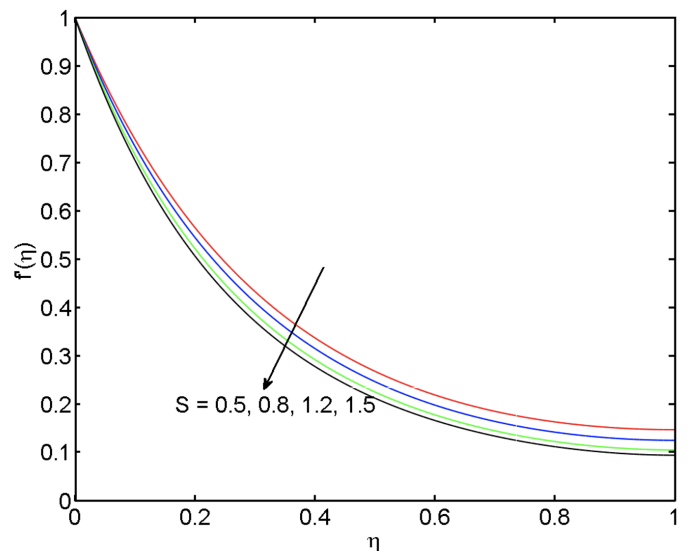

Fig. 3: Variations $S$ to $f^{\prime}$

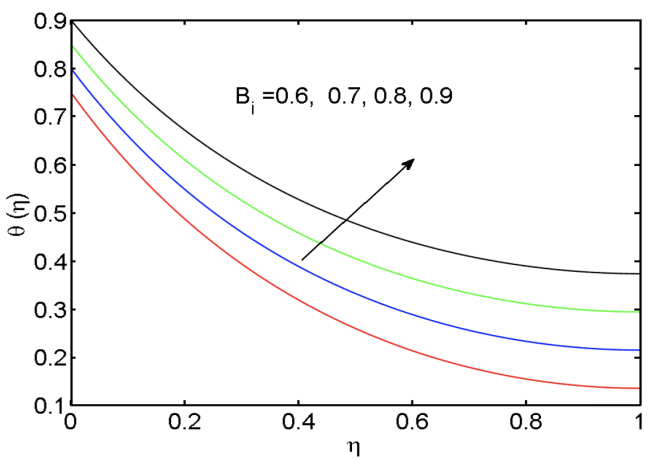

Fig. 5: Variations $B i$ to $\theta$

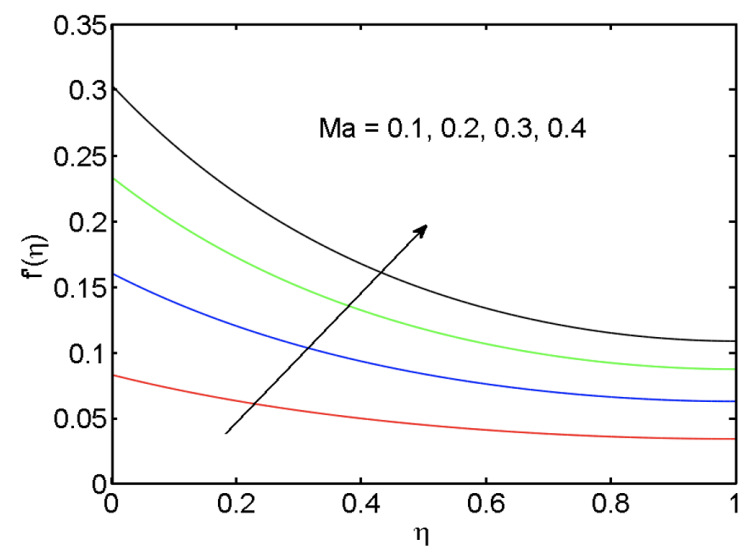

Fig. 6: Variations $M a$ to $f^{\prime}$ 
Table 2: The tabulation of numerical results for rate of heat transfer against the various values of rising parameters.

\begin{tabular}{|l|l|l|l|}
\hline$M$ & $\gamma$ & $B$ & $N u e_{x}^{-1 / 2}$ \\
\hline 0.5 & & & 1.813021 \\
\hline 1.0 & & & 1.803188 \\
\hline 1.5 & & & 1.792110 \\
\hline 2 & & & 1.638761 \\
\hline & 0.00 & & 1.802971 \\
\hline & 30 & & 1.790011 \\
\hline & 45 & & 1.775321 \\
\hline & 60 & & 1.764001 \\
\hline & & 0.5 & 1.813021 \\
\hline & & 1.0 & 1.739354 \\
\hline & & 1.5 & 1.529211 \\
\hline & & 2.0 & 1.375531 \\
\hline
\end{tabular}

Table 3: Thermophysical characteristics of kerosene oil and Copper

\begin{tabular}{|l|l|l|}
\hline Physical Characteristic & Kerosene Oil & $\mathrm{Cu}$ \\
\hline$\sigma(S / m)$ & 0.0042 & $5.96 \times 107$ \\
\hline$\rho\left(K g / m^{3}\right)$ & 997.1 & 8933 \\
\hline$C_{p}(J / K g K)$ & 4179 & 338 \\
\hline$k(w / m K)$ & 0.613 & 400 \\
\hline
\end{tabular}

\section{Conclusion}

In this study heat variation of nanofluid thin film flow is analyzed accompanied by heat source/sink and inclined magnetic field with viscous dissipation. With the above discussion subsequent conclusions are summarized as follows:

-The nanoparticle concentration and inclined magnetic field control the fluid velocity which causes enrichment in temperature in the thin-film flow.

-Both the temperature and velocity profiles decline for escalating values of the unsteadiness parameter.

-Velocity reduces for greater estimates of volume fraction of nanofluid and magnetic field whereas these parameters show an opposite behavior for temperature profile.

\section{Competing interests}

The authors declare that they have no competing interests.

\section{Authors' contributions}

All authors have contributed to all parts of the article. All authors read and approved the final manuscript.

\section{References}

[1] Sheikholeslami, M., Ganji, D. D., Gorji-Bandpy, M., \& Soleimani, S. (2014). Magnetic field effect on nanofluid flow and heat transfer using KKL model. Journal of the Taiwan Institute of Chemical Engineers, 45, 795-807.

[2] Khan, M., Malik, M. Y., Salahuddin, T., \& Hussian, A. (2018). Heat and mass transfer of Williamson nanofluid flow yield by an inclined Lorentz force over a nonlinear stretching sheet. Results in Physics, 8, 862-868. 
[3] Emslie, A.C.; Bonner, F.D.; Peck, L.G. Flow of a viscous liquid on a rotating disk. Journal of Applied Physics, 1958, $29,858-862$. [4] Al-Mdallal, Q. M., Indumathi, N., Ganga, B., \& Hakeem, A. A. (2020). Marangoni radiative effects of hybrid-nanofluids flow past a permeable surface with inclined magnetic field. Case Studies in Thermal Engineering, 17, 100571.

[5] Rasool, G., Shafiq, A., Khalique, C. M., \& Zhang, T. (2019). Magnetohydrodynamic Darcy-Forchheimer nanofluid flow over a nonlinear stretching sheet. Physica Scripta, 94, 105221.

[6] Sheikholeslami, M., \& Ganji, D. D. (2017). Influence of magnetic field on CuO-H2O nanofluid flow considering Marangoni boundary layer. International journal of Hydrogen energy, 42, 2748-2755. 